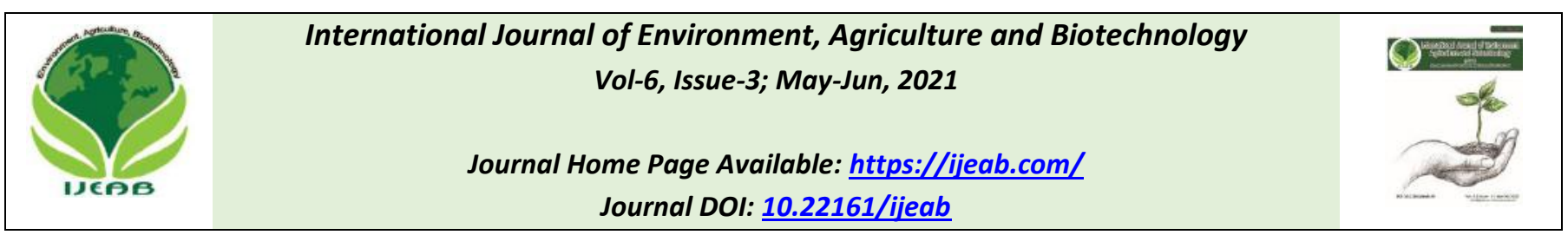

Article

\title{
Fungal Laccase as a Green Catalyst
}

\author{
Ram Sahay
}

Department of Chemistry, Maharana Pratap P. G. College, Jungle Dhusan, Gorakhpur, India

Received: 26 Feb 2021; Received in revised form: 25 Apr 2021; Accepted: 15 May 2021; Available online: 28 May 2021

(C)2021 The Author(s). Published by Infogain Publication. This is an open access article under the CC BY license

(https://creativecommons.org/licenses/by/4.0/).

\begin{abstract}
Laccases belongs to multinuclear copper containing oxidase and can act as a variety of aromatic and non-aromatic compounds. Due to their broad substrate specificity, they are considered as a promising candidate in various industrial and biotechnological sectors. They are regarded as a green catalyst in biotechnology. The present review focuses on structure, reaction mechanism, categories, applications, economic feasibility, limitations, and future prospects of fungal laccases. Thus, this review would help in understanding laccases along with the areas, whichhas not been focused and requires attention. Since past, immense work has been carried out on laccases: yet, new discoveries and application are ever increasing which includes biofuel, biosensor, fiber board synthesis, bioremediation, clinical, textile industry, food, cosmetics, and many more.
\end{abstract}

Key words-Bioremediation, Catalyst, Mediator, Oxidoreductase, Multicopper, Antibiotics.

\section{INTRODUCTION}

Laccases are p-diphenol:dioxygen oxidoreductase [EC 1.10.3.2] belonging to the family of multicopper proteins. It was first isolated from the sap of Japanese lacquer tree Rhus vernicifera [1] and are widely distributed in nature being found in plants,

Fungi [2-4] insects and bacteria first bacterial laccase was discovered in Azospirillum lipoferum. Since bacterial laccases have low redox potential [5] fungal laccases [6] are more preferred owing to their high redox potential [7-9]. Laccases have the ability to oxidize a wide range of aromatic and non-aromatic compounds which includes substituted phenols, some inorganic ions, and variety of non phenolic compounds [10] Due to its low substrate specificity it can act on a broad range of substrates and has attracted considerable attention in different environmental, industrial and biotechnological sectors [11-12]. Laccases have been regarded as a Green Tool, because they require molecular oxygen $\left(\mathrm{O}_{2}\right)$ as the only co-substrate for bio-catalysis and not hydrogen peroxide $\mathrm{H}_{2} \mathrm{O}_{2}$. Laccases have the capability to reduce dioxygen to water by oneelectron oxidation of substrate [13] which is mainly substituted phenolic compounds.

Laccases have high catalytic efficiency and are used for technical applications in various industrial and biotechnological domains [14-15] which includes improving properties of fibers, biosynthesis, energy exploitation, environmental protection, biodetection, degradation of synthetic dyes, printing and dyeing industry, biopulping in paper industry, conversion of aromatic compounds [16] and removal of phenols which causes cancer and teratogenicity when present in waste water [17]. In addition, it is also used in fast moving consumer goods as toothpaste, mouthwash, detergent, soap, and diapers in cosmetics as deodorants; in beverage and food industry for wine and juice stabilization [18-19] in dough or baked products to increase strength of gluten structures in pharmaceutical industries as anesthetics, anti inflammatory drugs, antibiotics, and sedatives and in nanobiotechnology as nanoparticle based biosensors. However, laccases are not able to exhibit full efficiency under harsh conditions. Therefore, novel strains which can tolerate harsh conditions and give maximum enzyme production with minimum energy consumption are in huge demand.

The present review is an attempt to provide cumulative information on various aspects of fungal laccases, which includes information pertaining to the structure, reaction mechanism,categories, and industrial and biotechnological 
application of laccase.

\section{SRUCTURE AND REACTION MECHANISM OF LACCASE}

Laccases are known to exist in a variety of forms; they can be monomeric, homotetrameric, heterodimeric, and multimeric. Their molecular weight ranges from 50 to 130 $\mathrm{kDa}$ depending upon the organism [20]. The plant laccases approximately contains $45 \%$ carbohydrate content, whereas for fungal laccases, it is $10-30 \%$ of molecular weight [21]. It is assumed that the carbohydrate portion of laccase ensures the conformational stability of the protein part and protects the enzyme from proteolysis and inactivation by radicals. The primary structure of laccases consists of Greek key $\beta$ barrel topology which constitutes of approximately 500 amino acid residues organized in three consecutive domains. These amino acids are distributed in three domains: first domain with 150 initial amino acids, second domain with 150 and 300 amino acid, and third domain with 300-500 amino acids. The stabilization of laccase structure is due to the presence of disulphide bonds between domains I and II and between domains I and III [22]. However, in Melanocarpus albomyces, three disulfide bridges are present of which one is inside domain I, another between domain I and domain III and the last one between domain II and III [23]. Laccases are known to exist in four different $\mathrm{Cu}$ catalytic forms per protein unit. These four $\mathrm{Cu}$ ions are divided into three types of structures:

\section{TYPE-1: PARAMAGNETIC BLUE COPPER}

Copper type-1 confers blue color to multicopper proteins, which is due to the intense electronic absorption caused by the covalent copper cysteine bond. Due to its high redox potential of $+790 \mathrm{mV}$ substrate oxidation takes place at the type-1 copper site and has an absorbance at $610 \mathrm{~nm}$ type-1 copper has a trigonal coordination, with two histine and a cysteine as conserved equatorial ligands and one position usually variable, and in case of fungal laccases, the axial ligand is leucine or phenylalanine. It has even been argued that the axial position ligand influences the oxidation potential of the enzyme, which possibly provides the mechanism for regulating its activity [24].

TYPE-2: PARAMAGNETIC NORMAL/NON-BLUE COPPER
Copper tpe- 2 or normal $\mathrm{Cu}$ site is characterized by the lack of strong absorption features in the visible region and reveals usual electron paramagnetic resonance (EPR) spectra. Type- 2 copper is coordinated by two histidines residues and is strategically positioned close to type- 3 copper.

TYPE-3: DIAMAGNETIC SPIN COUPLED COPPERCOPPER PAIR

It is a binuclear center regulated by six histidines and spectroscopically characterized by an electron adsorption at $330 \mathrm{~nm}$ oxidized form and absence of an EPR signal due to the strong antiferromagnetical coupling between the two type- 3 copper atoms which is related to the presence of a hydroxyl bridge. The type- 2 copper and type-3 copper form a trinuclear cluster where molecular oxygen is reduced and release of water takes place. An example of one electron oxidation of phenolic hydroxyl groups, while reducing oxygen and forming phenoxy radicals along with water are represented below [25]:

$$
4 \mathrm{Phe}-\mathrm{OH}+\mathrm{O}_{2} \stackrel{\text { Laccas }}{\longrightarrow} 4 \mathrm{Phe}-\mathrm{O}^{\circ}+2 \mathrm{H}_{2} \mathrm{O}
$$

The Type- 3 copper centers also have common feature of another protein superfamily which includes the tyrosinases and haemocyanins [26] figure-1.

\section{REACTION MECHANISM OF LACCASE: DIRECT AND INDIRECT OXIDATION}

The basic reactions catalyzed by laccase can be of two types: direct oxidation and in direct oxidation. The direct oxidation involves the oxidation of substrate to the corresponding radical as a result of direct interaction that occurs with copper cluster. However, in certain reactions, direct oxidation is not feasible as laccase can only oxidize those compounds whose ionization potential does not exceed redox potential of T1 copper ion. Nevertheless, the limitation can be overcome by the use of mediator which is a two step process: first enzyme catalyzes the oxidation of the mediator and then the oxidized mediator oxidizes the substrate. However, for the reaction to occur without any obstruction, certain features should be exhibited by the mediator: (a) the reaction should occur without any hindrance it must be good substrate for laccase both in its oxidized and reduced forms, should be stable; it must not inhibit enzymatic reaction, and conversion must be cyclic in nature [27] shown in figure. 2 


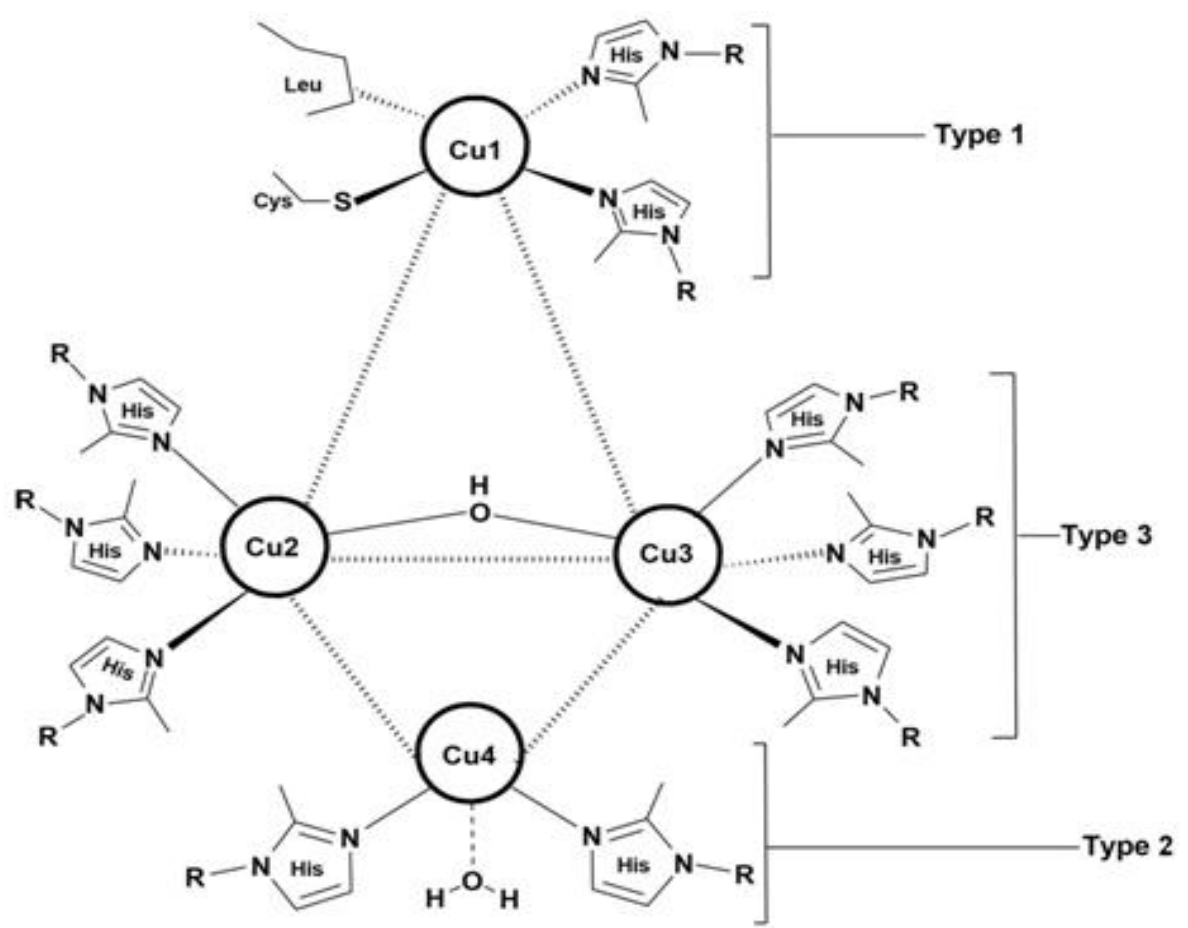

Fig: 1 Copper centers of laccase: Type-1, Type-2, and Type-3 (Adapted and modified axial ligand as leucine as per fungal laccases)

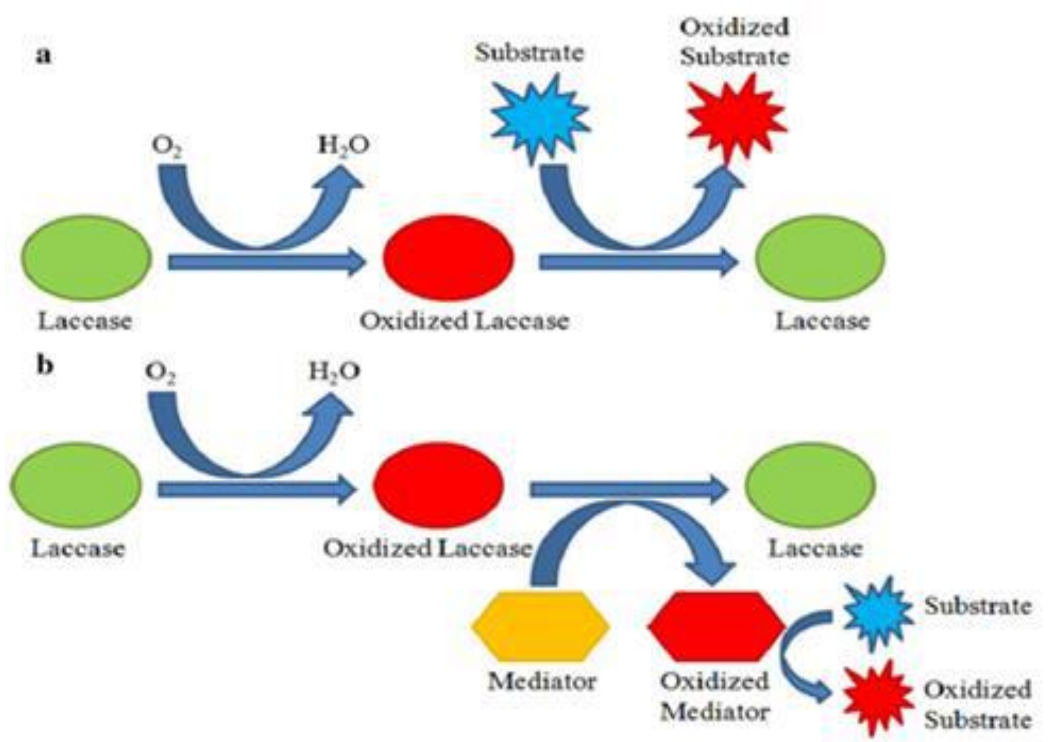

Fig: 2 Schematic representation of reaction catalyzed by laccase; a direct oxidation: the substrate is oxidized to the corresponding radical as a result of direct interaction and $b$ indirect oxidation: the substrate is oxidized in the presence of a mediator

Besides mediators, the use of inducer to enhance laccase production has been widely practiced in fungi especially in the white rots where metals, aromatic compounds, and phenolic compounds [28] have been used as inducers. Conversely, there are certain substances which can inhibit the production of laccase and are known as the inhibitors such as sodium azide and dithiothreitol DTT.

\section{YELLOW LACCASE}

Yellow laccase is artificially reduced blue laccase as it does not have absorption at $600 \mathrm{~nm}$ and EPR spectrum [29]. Alteration of yellow to blue laccase can occur by the reduction of type-I copper site by aromatic product of lignin degradation or binding of specific amino acid of enzyme polypeptide to a molecule of modified product 
produced by lignin degradation; it can also be due to heterogeneity induced by glycosylation. The modified molecule bound to the apoenzyme performs the function of electron transfer mediator analogous to the role of 2,2-azinobis (3-ethylbenzthiazoline -6-sulfonic acid) diammonium salt (ABTS) or other compounds in the reaction of blue laccase, hence having high redox potential which allows them to oxidize non phenolic compounds without any mediatorsand having greater industrial potential [30]. The change in protein conformation may explain the sensitivity of yellow laccase to $\mathrm{CO}$ and other inhibitors, e.g., P. tigrinus. At present, information about the purification and characterization of yellow laccase is extremely limited and their catalytic properties are still seldom investigated. Much work has not been done on the yellow laccase, but a few strains which are reported for the production of yellow laccase are as follows $P$. tigrinus [29], S. aeruginosa, G. fornicatum [30], P. ostreatus [31], Panustigrinus, Phlebia radiate, Phlebia tremellosa, Pleurotus ostreatus D1 (YLPO), Sclerotinia sclerotiorum, and Panustigrinus [30].

\section{WHITE LACCASE}

The white laccase exhibits neutral $\mathrm{pH}$ and has anomalous metal content which is responsible for its unique characteristic [32]. It exhibits absorption peak at $400 \mathrm{~nm}$ but absence of peak at $605 \mathrm{~nm}$ T1 copper site and $330 \mathrm{~nm}$ (T3 binuclear copper). White laccase does not exhibit EPR spectra as well as T1 and T2 signals [33]. The reason for the colorlessness iron, but it was conferred that the lack of absorbance at $605 \mathrm{~nm}$ can be due to the incomplete oxidation state of copper which has fully occupied electron configuration of $\mathrm{d} 10$ and no $\mathrm{d}-\mathrm{d}$ transition [33] and may be responsible for extra high activity of protein. White laccase has been considered under laccase family, because the primary structure of the white laccase is identical to those of known laccase and it uses oxygen $\left(\mathrm{O}_{2}\right)$ as an oxidative substrate. There is the absence of the formation of hydrogen peroxide $\left(\mathrm{H}_{2} \mathrm{O}_{2}\right)$ as the product of catalyzed reaction and substrate specificity exhibited is also the same as that of known laccase [32], e.g., Lepista nuda molecular mass of $56 \mathrm{kDa}$ [34].

Few strains reported for white laccase production include Pleurotus ostreatus [32] and Myrothecium verrucaria NF-05 [33]. These strains besides single copper atom consist of various.

\section{LACCASE MEDIATOR}

The efficiency of substrate oxidation by a laccase depends on the difference between the redox potentials [35] of the substrate and the $\mathrm{T} 1 \mathrm{Cu}$. Due to the lower redox potentials of laccases $(\leq 0.8 \mathrm{~V})$ compared to ligninolytic peroxidases $(>1 \mathrm{~V})$ [36] laccases are originally thought to be able to oxidize only the phenolic ligninmoiety, with the majority of lignin being nonphenolic and with higher redox potentials. Low molecular- weight redox mediators are used to expand the laccase substrate range or increase the reaction rate, especially for substrates with higher redox potentials or too large to fit in the enzyme's active site. Commonly used laccase mediators include synthetic mediators such as 2,2'-azino-bis-(3-ethylbenzothiazoline6-sulfonate) (ABTS) and 1-hydroxybenzotriazole (HBT) as well as natural phenolic mediators such as syringaldehyde and acetosyringone. Despite the proven efficiency of artificial mediators, natural mediators (believed to be true mediators of fungal laccases in nature) are considered to be alternatives to the artificial ones because they are more economically feasible and environmentally friendly. Laccase oxidation of the substrate may proceed differently with a mediator. However, it is not always the case. Malachite green degradation products in the presence and absence of ABTS have been shown to be identical or different, depending on the enzyme.

Different types of mediators have different catalytic mechanisms ABTS mediated substrate oxidation proceeds via an electron transfer route. ABTS is first oxidized to its radical cation $\left(\mathrm{ABTS}^{+}\right)$and then to the dication $\left(\mathrm{ABTS}^{2+}\right)$ with redox potentials of 472 and $885 \mathrm{mV}$, respectively. Unlike ABTS, an N-OH type mediator (such as HBT and violuric acid) forms the $\mathrm{N}$-oxy radical upon laccase oxidation and subsequent deprotonation the radical in turn abstracts the benzylic hydrogen atom from the substrate. Similarly, phenolic mediators also follow a radical hydrogen abstraction mechanism, but with the intermediate being a phenoxy radical. The effect of a mediator on laccase oxidation varies with the laccase and substrate and depends on the radicals formed, recyclability of the mediator and stability of the laccase in the presence of the mediator [37]. Regardless of the reaction mechanism, mediators incur additional costs, and can cause toxicity [38] and laccase inactivation [39]. Although, laccases without the requirement for facilitating mediators, the laccase/mediator system is regarded as a feasible industrial solution, ideal mediators that are cheap, green, effective, stable, recyclable, not toxic, or enzyme-inactivating should be ascertained [40].

\section{LACCASE IMMOBILIZATION}

Laccases are immobilized for recycling, operational stability, and resistance to application conditions. Immobilization techniques include entrapment, adsorption, covalent binding, self immobilization as well as combinations of the aforementioned techniques. Activity recovery varies based on the enzyme, the 
immobilization method of choice, and preparation parameters. Compared with their free counterparts, immobilized laccases are more tolerant to high temperatures and storage and can be reused multiple times [41-42] they are also more resistant to inhibitors such as $\mathrm{NaCl}$. Immobilization sometimes improves the catalytic activity of laccases [43-45] despite the common concern of reduced enzyme flexibility, steric hindrance and diffusion limitations [46]. Readers can refer to reviews on preparation and applications of immobilized laccases [4142].

\section{LACCASE APPLICATIONS IN BIODEGRADATION OF PPCPS}

The value of fungi as well as fungal enzymes in pollution control and environment management has been recognized. Examples of environmentally important enzymes comprise hydrolases, laccases, lyases, peroxidases, tyrosinases, and $\mathrm{P} 450$ cytochrome mono oxidases [47-50]. The ability of laccases to effectively degrade and detoxify a variety of persistent organic pollutants (POPs) has received considerable attention in the field of bioremediation [51-53] and laccases can also be used in enzymatic biosensors for environmental pollution monitoring [48]. A summary of environmental contaminants as laccase substrates is provided in (Table1). The contaminants investigated include dye stuffs [5455], polycyclic aromatic hydrocarbons (PAHs) [56], endocrine disrupters [51, 57] and pesticides [58] shown in figure-3.

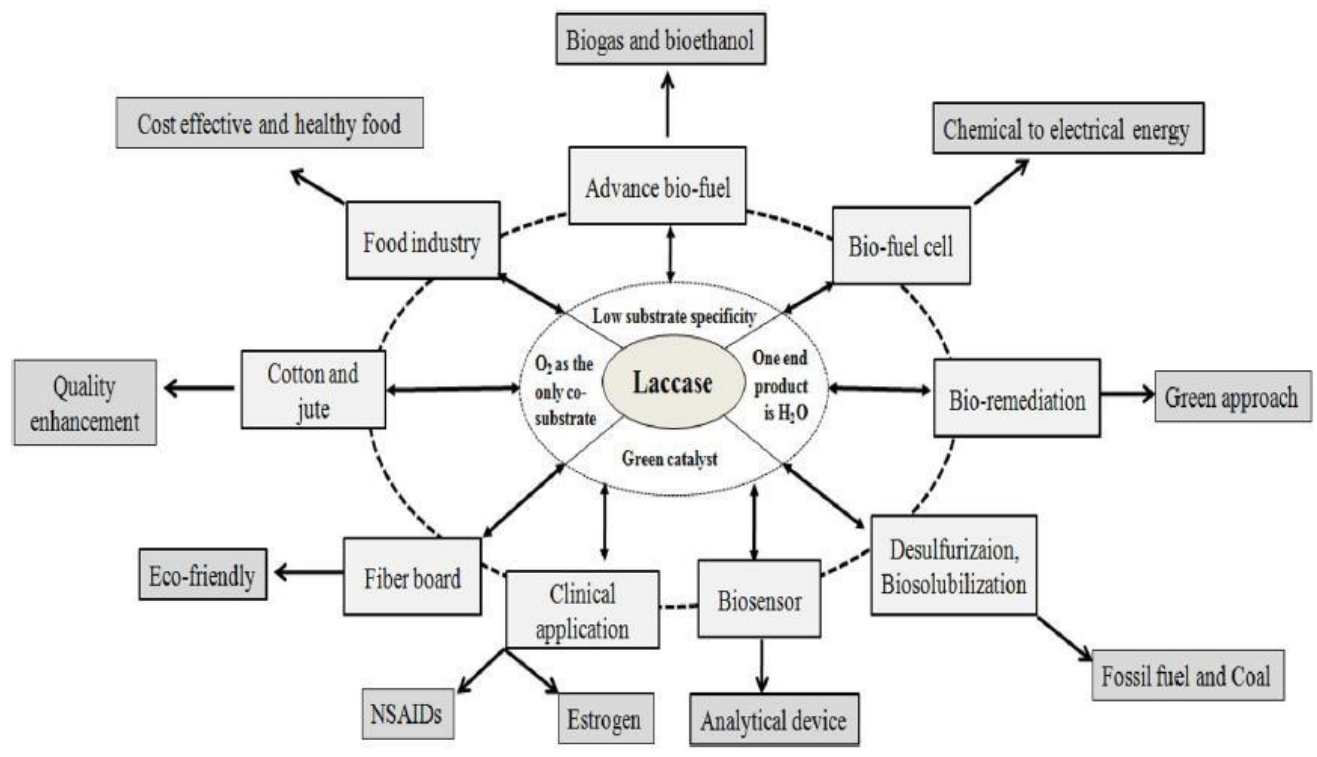

Fig: 3 Applications of laccase in various industries and biotechnological sectors

Pharmaceuticals and personal care products (PPCPs) are becoming ubiquitous in the environment and are recognized as emerging trace organic contaminants [5961]. Laccases can be employed for their removal [51]. Laccases have been used in PPCPs as an ingredient; many products generated by laccases have antimicrobial, anticancer, antioxidant, detoxifying, or other activities [62]. Specifically, laccases can be used to synthesize novel antibiotics and laccase based antimicrobial formulations are considered as a green alternative to chemical decontamination. Nonetheless, the focus of this review lies in the degradation and detoxification of PPCPs contaminants with laccases.

\section{DEGRADATION OF ANTIBIOTICS}

Antibiotics constitute one of the most used classes of drugs in the world; they are used in human and veterinary medicine as well as livestock farming. Antibiotics that are not metabolized enter the environment. Conventional water treatment processes cannot effectively remove antibiotics [61], while more efficient advanced treatment methods have disadvantages such as high costs and secondary pollution. Antibiotics pose health risks by selecting for antibiotic resistance bacteria (ARB). Antibiotics, ARB, and antibiotic resistant genes have been detected in soil, sediments, and water bodies including waste water drinking water and marine water. There has been a fast growth in the literature describing laccase 
utilization in antibiotic removal, especially within the past 2 years, but this topic has not been properly reviewed.

Target antibiotics under investigation include penicillins, tetracyclines, sulfonamides, uinolones, trimethoprim, sulfamethoxazole and tetracycline are two most studied (Table-2). The removal time ranges from minutes to hours, depending on the laccase, antibiotic and treatment parameters. Mediators such as HBT, ABTS, and SA are often used to enable or accelerate antibiotic conversion by laccases. Infact, significant antibiotic removal within $1 \mathrm{hrs}$ usually requires involvement of an appropriate mediator [63]. Manganese peroxidase was more efficient in tetracycline conversion than laccase, but the addition of HBT can promote laccase catalysis to a rate higher than that of manganese peroxidase although still slower than that of lignin peroxidase (95\%) degradation efficiency in $5 \mathrm{~min}$. Interestingly, mediators, i.e., ABTS, SA, and AS, are consumed without observed catalytic activity during degradation of sulfamethoxazole [64]. Sulfonamides and tetracyclines are more easily attacked by laccase compared with quinolones [65].

Table1. Laccase Applications in Biodegradation and Bioremediation

\begin{tabular}{|c|c|c|c|c|}
\hline Compound & Laccase & Enzyme From & Mediator & $\begin{array}{r}\text { Ref } \\
\text { erence }\end{array}$ \\
\hline \multicolumn{5}{|c|}{ Phenol } \\
\hline $\begin{array}{l}\text { Chlorophenols, cresols, } \\
\text { nitrophenols }\end{array}$ & $\begin{array}{l}\text { Trametessanguineu } \\
\text { slaccase expressed in } \\
\text { Trichoderma atroviride }\end{array}$ & In culture & - & [54] \\
\hline Technicalnonylphenol & $\begin{array}{l}\text { Phomasp. UHH5- } \\
\text { 1-03 }\end{array}$ & Free & SA & [76] \\
\hline $\begin{array}{c}\text { Oxybenzone, } \\
\text { pentachlorophenol }\end{array}$ & P.ostreatus & Free & $\begin{array}{l}\text { ABTS, HBT, HPI, } \\
\text { TEMPO, SA, VA, } \\
\text { VAN }\end{array}$ & [30] \\
\hline $\begin{array}{l}\text { 4-tert-butylphenol,4-tert- } \\
\text { octylphenol }\end{array}$ & $\begin{array}{l}\text { Myceliophthorather } \\
\text { mophila laccase } \\
\text { expressed in } \\
\text { Aspergillus oryzae } \\
\text { (Novozyme) }\end{array}$ & $\begin{array}{c}\text { Enzymatic } \\
\text { membranereactor }\end{array}$ & SA & [72] \\
\hline 2,4-dichlorophenol & $\begin{array}{r}\text { Pycnoporuss } \\
\text { anguineus CS43 }\end{array}$ & Free & - & [37] \\
\hline \multicolumn{5}{|c|}{ DYESTUFFS } \\
\hline $\begin{array}{l}\text { Bromo phenol Blue, Congo } \\
\text { Red, Coomassie Blue, Tripan } \\
\text { Blue }\end{array}$ & $\begin{array}{c}\text { T. sanguineus } \\
\text { laccase expressed in } \\
T . \text { atroviride }\end{array}$ & Free & - & [54] \\
\hline $\begin{array}{l}\text { Acid Black 172, Congo Red, } \\
\text { Crystal Violet, Direct Fast Blue } \\
\text { FBL, Indigo Blue, Naphthol } \\
\text { Green B, Methylene Blue, } \\
\text { Neutral Red, Reactive Brilliant } \\
\text { BlueX-BR, Remazol Brilliant } \\
\text { Blue Reactive (RBBR) }\end{array}$ & T. pubescens & Chitosanbeads & - & [10] \\
\hline $\begin{array}{c}\text { Acid Orange 67, Basic } \\
\text { Red18, } \\
\text { Basic Yellow 28, Direct } \\
\text { Black166, Direct Yellow107, } \\
\text { Disperse Yellow } 79\end{array}$ & $\begin{array}{l}\text { Paraconiothyrium } \\
\text { variabile }\end{array}$ & Free & HBT & [64] \\
\hline
\end{tabular}




\begin{tabular}{|c|c|c|c|c|}
\hline $\begin{array}{c}\text { Brilliant Blue-G, Brilliant } \\
\text { Blue-R, Bromo phenol Blue, } \\
\text { Coomassie Blue R250, Crystal } \\
\text { Violet, Malachite Green, } \\
\text { Methylene Blue, Xylene Cyanol, } \\
\text { RBBR }\end{array}$ & P.sanguineus & Free & VA & [30] \\
\hline RBBR & Cerrenasp.HYB07 & $\begin{array}{l}\text { Cross-linked enzyme } \\
\text { aggregates }\end{array}$ & - & [43] \\
\hline Coomassie Blue-R 250 & Cerrenasp. HYB07 & $\begin{array}{l}\text { Cross-linked enzyme } \\
\text { aggregates }\end{array}$ & $\begin{array}{l}\text { ABTS, AS, HBT, } \\
\text { SA, SYA }\end{array}$ & [73] \\
\hline \multicolumn{5}{|c|}{ ENDOCRINE DISRUPTERS } \\
\hline Bisphenol-A (BPA) & $\begin{array}{l}\text { Coriolopsis gallica, } \\
\text { Bjerkandera adusta, } T . \\
\text { versicolor }\end{array}$ & Free & HBT & [38] \\
\hline BPA & $\begin{array}{c}\text { T.sanguineus } \\
\text { laccase expressed in } \\
\text { T. atroviride }\end{array}$ & In culture & - & [54] \\
\hline BPA & $\begin{array}{l}\text { T.versicolor laccase } \\
\text { expressed in } \\
\text { S.Cerevisiae }\end{array}$ & Surface display & ABTS & [61] \\
\hline BPA & $\begin{array}{l}\text { M.thermophila } \\
\text { laccase expressed in } \\
\text { A. oryzae } \\
\text { (Novozyme) }\end{array}$ & $\begin{array}{l}\text { Ongranular activated } \\
\text { carbon, continuous flow } \\
\text { packed-bed reactor }\end{array}$ & - & [72] \\
\hline BPA, $17 \alpha$-ethinyl estradiol & T.versicolor & $\begin{array}{c}\text { Polyamide- } 6 \\
\text { /chitosannao fibers }\end{array}$ & - & [72] \\
\hline BPA, 17 $\alpha$-ethinyl estradiol & $\begin{array}{l}\text { Phomasp. UHH5-1- } \\
03\end{array}$ & Free & SA & [77] \\
\hline $\begin{array}{l}\text { BPA, } 17 \alpha \text {-ethinyl estradiol, } \\
\text { 17 } \alpha \text {-estradiol, } 17 \alpha \text {-estradiol 17- } \\
\text { acetate, estriol, estrone }\end{array}$ & $\begin{array}{c}\text { M.thermophila } \\
\text { laccase expressed in } \\
\text { A. oryzae } \\
\text { (Novozyme) }\end{array}$ & $\begin{array}{l}\text { Enzymatic membrane } \\
\text { reactor }\end{array}$ & SA & {$[72]$} \\
\hline \multicolumn{5}{|c|}{ POLYCYCLIC AROMATIC HYDROCARBONS (PAHs) } \\
\hline All-15 USEP ApriorityPAHs & $\begin{array}{c}\text { B.subtilis Cot } \\
\text { Aexpressedin E.coli }\end{array}$ & Free & ABTS & [56] \\
\hline Naphthalene, phenanthrene & $\begin{array}{l}\text { T.versicolor (Sigma- } \\
\text { Aldrich) }\end{array}$ & $\begin{array}{l}\text { Non ionic surfactant- } \\
\text { modified clay }\end{array}$ & - & {$[56]$} \\
\hline $\begin{array}{l}\text { Benzo [a] pyrene, } \\
\text { phenanthrene }\end{array}$ & $\begin{array}{c}\text { T.sanguineus } \\
\text { laccase expressed in } \\
\text { T. atroviride }\end{array}$ & Free & - & [54] \\
\hline \multicolumn{5}{|c|}{ PESTICIDES } \\
\hline Atrazine & P.ostreatus & Free & $\begin{array}{l}\text { ABTS, HBT, } \\
\text { HPI, TEMPO, SA, } \\
\text { VA,VAN }\end{array}$ & [30] \\
\hline Atrazine, isoproturon & $\begin{array}{c}\text { O.sativa laccases } \\
\text { expressed in P.pastoris }\end{array}$ & Inculture & - & [58] \\
\hline
\end{tabular}




\begin{tabular}{|c|c|c|c|}
\hline $\begin{array}{c}\text { Ametryn, atrazine, clofibric } \\
\text { acid, fenoprop, pentachloro } \\
\text { phenol, propoxur }\end{array}$ & $\begin{array}{c}\text { M.thermophilal } \\
\text { accase expressed in }\end{array}$ & $\begin{array}{c}\text { Enzymatic membrane } \\
\text { reactor } \\
\text { A. oryzae } \\
\text { (Novozyme) }\end{array}$ & SA \\
\hline
\end{tabular}

Table:2 Laccase Treatments of Antibiotics

\begin{tabular}{|c|c|c|c|c|c|c|}
\hline Compound & Laccase & $\begin{array}{l}\text { Enzyme } \\
\text { from }\end{array}$ & $\begin{array}{l}\text { Reaction } \\
\text { parameters }\end{array}$ & Efficiency & $\begin{array}{l}\text { Toxicity after } \\
\text { treatment }\end{array}$ & $\begin{array}{l}\text { Referen } \\
\text { ces }\end{array}$ \\
\hline \multicolumn{7}{|c|}{ PENICILLINS } \\
\hline $\begin{array}{l}\text { Amoxicillin } \\
\text {, ampicillin, } \\
\text { cloxacillin, } \\
\text { penicillin-G, } \\
\text { penicillinV, } \\
\text { oxacillin }\end{array}$ & $\begin{array}{c}T . \\
\text { versicolor } \\
\text { (Sigma- } \\
\text { Aldrich) }\end{array}$ & $\begin{array}{l}\text { Enzymatic } \\
\text { membrane } \\
\text { reactor }\end{array}$ & $\begin{array}{l}10 \mu \mathrm{g} / \mathrm{L} \text { antibiotics, } 1 \mathrm{~m} \\
\mathrm{MSA}, \text { starting } \mathrm{pH} 6, \\
25^{\circ} \mathrm{C}, 0.07 \mathrm{~m} / \mathrm{s} \text { flow, } \\
\text { tangential configuration }\end{array}$ & $\begin{array}{l}54-100 \% \\
\text { after } 24 \mathrm{~h}\end{array}$ & $\begin{array}{c}\text { Increased } \\
\text { (B.subtilisand } V . \\
\text { fischeri) }\end{array}$ & [39] \\
\hline \multicolumn{7}{|c|}{ SULFONAMIDES } \\
\hline $\begin{array}{l}\text { Sulfapyridine, } \\
\text { sulfathiazole }\end{array}$ & $\begin{array}{l}\text { T.versicolor } \\
\text { (Sigma- } \\
\text { Aldrich) }\end{array}$ & Free & $\begin{array}{l}\text { 16-20 mg/L antibiotic, } \\
\text { 50-55 U/L laccase, } 0.8 \\
\mathrm{mM} \mathrm{VA,} \mathrm{pH} 4.5,25^{\circ} \mathrm{C} \\
135 \mathrm{rpm}\end{array}$ & $\begin{array}{l}100 \% \text { after } \\
8 \mathrm{~h}\end{array}$ & NR & [74] \\
\hline $\begin{array}{l}\text { Sulfadimet } \\
\text { hoxine, } \\
\text { sulfamonometh } \\
\text { oxine }\end{array}$ & $\begin{array}{r}\text { Perenni } \\
\text { poriastrain } \\
\text { TFRI } 707\end{array}$ & Free & $\begin{array}{l}50 \mathrm{mg} / \mathrm{L} \text { antibiotic, } 6 \\
\mathrm{U} / \mathrm{mL}, 1 \mathrm{mM} \text { ABTS or } \\
\mathrm{VA}, \mathrm{pH} 4.1,30^{\circ} \mathrm{C}, 8 \% \\
\text { glycerol }\end{array}$ & $\begin{array}{c}T(1 / 2) \\
(\min ): 1.8-4.1\end{array}$ & NR & [79] \\
\hline $\begin{array}{l}\text { Sulfadimet } \\
\text { hoxine, } \\
\text { sulfamonometh } \\
\text { oxine }\end{array}$ & $\begin{array}{r}\text { Perenni } \\
\text { poriastrain } \\
\text { TFRI } 707\end{array}$ & Free & $\begin{array}{c}50 \mathrm{mg} / \mathrm{L} \text { antibiotic, } \\
6 \mathrm{U} / \mathrm{mL} \\
\text { laccase, } 8 \% \text { glycerol; } \\
1 \mathrm{mM} \text { ABTS, pH 4, 50- } \\
60^{\circ} \mathrm{C} ; 1 \mathrm{mM} \mathrm{VA}, \mathrm{pH} 4,40- \\
60^{\circ} \mathrm{C} ; 2 \mathrm{~m} \mathrm{MSA}, \mathrm{pH} 6 \\
50^{\circ} \mathrm{C}\end{array}$ & $\begin{array}{l}100 \% \text { after } \\
30 \text { min with } \\
\text { ABTS; } \\
100 \% \text { after } 15 \\
\text { min } \\
\text { withVA; } \\
>95 \% \text { after } 60 \\
\text { min with SA }\end{array}$ & $\begin{array}{c}\text { Reduced } \\
(V . \text { fischeri }) \\
\text { withVA and } \\
\text { HBA; increased } \\
\text { with ABTS } \\
\text { andSA }\end{array}$ & [40] \\
\hline $\begin{array}{l}\text { Sulfametho } \\
\text { xazole }\end{array}$ & $\begin{array}{l}\text { T.versicolo } \\
r\end{array}$ & Free & $\begin{array}{r}1,100 \mu \mathrm{g} / \mathrm{L} \text { antibiotic, } \\
1 \mathrm{mM} \mathrm{HBT}, 25^{\circ} \mathrm{C}, 70 \mathrm{rpm}\end{array}$ & $\begin{array}{l}41 \% \text { after } \\
22 \mathrm{~h}\end{array}$ & NR & {$[48]$} \\
\hline $\begin{array}{l}\text { Sulfametho } \\
\text { xazole }\end{array}$ & $\begin{array}{l}\text { M.thermop } \\
\text { hila laccase } \\
\text { expressed } \\
\text { in A.oryzae } \\
\text { (Novozyme } \\
\text { ) }\end{array}$ & $\begin{array}{l}\text { Enzymati } \\
\text { c membrane } \\
\text { reactor }\end{array}$ & $\begin{array}{c}830 \mu \mathrm{g} / \mathrm{L} \mathrm{d} \text { antibiotic, } \\
70-100 \\
\mu \mathrm{M} / \mathrm{min} \text { laccase, } 5 \mu \\
\mathrm{MSA}, 3 \mathrm{~g} / \mathrm{L} \text { granular } \\
\text { activated carbon }\end{array}$ & $65 \%$ & $\begin{array}{l}\text { Increased (Tox } \\
\text { Screen3 assay with } \\
\text { Photobacterium } \\
\text { leiognathi), which } \\
\text { can be reduced by } \\
\text { granular activated } \\
\text { carbon addition }\end{array}$ & [69] \\
\hline $\begin{array}{r}\text { Sulfadiazine } \\
\text {,sulfamethazin, } \\
\text { sulfamethoxaz }\end{array}$ & $\begin{array}{c}\text { Echinod } \\
\text { ontiumtaxo } \\
\text { dii }\end{array}$ & $\begin{array}{l}\text { Oriented } \\
\text { immobilizati } \\
\text { onon }\end{array}$ & $\begin{array}{c}50 \mathrm{mg} / \mathrm{L} \text { antibiotic, } \\
0.2 \mathrm{U} / \mathrm{mL} \text { laccase, } 1 \mathrm{~m} \\
\text { MAS, SA or SYA, pH5 }\end{array}$ & $\begin{array}{l}>95 \% \text { after } \\
30 \mathrm{~min}\end{array}$ & $\begin{array}{c}\text { Reduced } \\
\text { (E.coliandS. } \\
\text { aureus })\end{array}$ & [67] \\
\hline
\end{tabular}




\begin{tabular}{|c|c|c|c|c|c|c|}
\hline ole & & $\begin{array}{c}\mathrm{Fe} 3 \mathrm{O} 4 \\
\text { nanoparticle } \\
\mathrm{s}\end{array}$ & & & & \\
\hline $\begin{array}{l}\text { Sulfametho } \\
\text { xazole }\end{array}$ & $\begin{array}{c}\text { T. } \\
\text { versicolor } \\
\text { (Sigma- } \\
\text { Aldrich) }\end{array}$ & Free & $\begin{array}{c}\text { 73-93 } \mu \text { Mantibiotic, } \\
\text { mediator/laccase ratio: } \\
1.1 \text { (ABTS), } 1.7(\mathrm{SA}) \text { or } \\
2.4(\mathrm{AS}), \mathrm{pH} \\
6,25^{\circ} \mathrm{C} \text {, static }\end{array}$ & $100 \%$ & $\begin{array}{c}\text { Reduced } \\
\text { (algae } \\
\text { Pseudokirchneri } \\
\text { ellasubcapitata) }\end{array}$ & {$[68]$} \\
\hline $\begin{array}{l}\text { Sulfametho } \\
\text { xazole, } \\
\text { sulfathiazole }\end{array}$ & $\begin{array}{l}\text { T.versic } \\
\text { olor }\end{array}$ & $\begin{array}{c}\text { On } \\
\text { poroussilica } \\
\text { beads }\end{array}$ & $\begin{array}{c}50 \mathrm{mg} / \mathrm{L} \text { antibiotic, } \\
1 \mathrm{U} / \mathrm{mL} \\
\text { laccase, } 1 \mathrm{mMHBT}, \mathrm{pH} \\
5,40^{\circ} \mathrm{C}, 50 \mathrm{rpm}\end{array}$ & $\begin{array}{l}76-85 \% \\
\text { after1h }\end{array}$ & $\begin{array}{c}\text { Reduced }(E . \\
\text { coli, } P . \\
\text { aeruginosa, } H . \\
\text { influenza,S. } \\
\text { enterica, } S . \\
\text { aureus, } S . \\
\text { pneumoniae })\end{array}$ & [75] \\
\hline $\begin{array}{l}\text { Sulfadimet } \\
\text { hoxine }\end{array}$ & $\begin{array}{l}\text { T.versic } \\
\text { olor }\end{array}$ & Free & $\begin{array}{l}\text { Pergram soil: } 2 \mu \mathrm{g} \\
\text { antibiotic, } 10 \mathrm{U} \text { laccase, } \\
8 \mu \mathrm{mol} \text { ABTS or HBT, } \\
\text { 1mg peat; room } \\
\text { temperature }\end{array}$ & $\begin{array}{l}>90 \% \text { after } \\
72 \mathrm{~h}\end{array}$ & NR & [78] \\
\hline $\begin{array}{l}\text { Sulfametho } \\
\text { xazole }\end{array}$ & $\begin{array}{c}T \\
\text { versicolor } \\
\text { Lac3 } \\
\text { expressed } \\
\text { in } S . \\
\text { cerevisiae }\end{array}$ & $\begin{array}{l}\text { Surfaced } \\
\text { isplay }\end{array}$ & $\begin{array}{c}30 \mu \mathrm{M} \text { antibiotic, } 0.25 \\
\mathrm{U} / \mathrm{mL} \text { laccase, } \mathrm{pH} 5,37^{\circ} \mathrm{C}, \\
250 \mathrm{rpm}\end{array}$ & $\begin{array}{l}44 \% \text { after } \\
30 \mathrm{~h}\end{array}$ & NR & [61] \\
\hline \multicolumn{7}{|c|}{ TETRACYCLINES } \\
\hline Tetracycline & $\begin{array}{l}T . \\
\text { versicolor } \\
\text { (Sigma- } \\
\text { Aldrich) }\end{array}$ & $\begin{array}{l}\text { Enzymatic } \\
\text { membrane } \\
\text { reactor }\end{array}$ & $\begin{array}{c}20 \mathrm{mg} / \mathrm{L} \text { antibiotic, } \\
0.002 \mathrm{~g} / \mathrm{L} \text { laccase }, \mathrm{pH} \\
6,25^{\circ} \mathrm{C} \text {, batch }\end{array}$ & $\begin{array}{l}0.34 \mathrm{mg} / \mathrm{h} \\
\text { for } 10 \mathrm{~d}\end{array}$ & NR & [71] \\
\hline Tetracycline & $\begin{array}{c}\text { T. } \\
\text { versicolor } \\
\text { (Sigma- } \\
\text { Aldrich) }\end{array}$ & $\begin{array}{l}\text { Enzymati } \\
\text { c membrane } \\
\text { reactor }\end{array}$ & $\begin{array}{c}20 \mathrm{mg} / \mathrm{L} \text { antibiotic, } \\
10 \mathrm{~g} / \mathrm{L} \\
\text { laccase, } 1.4 \mu \mathrm{m} \\
\text { poresize, } 25 \mathrm{~cm} \\
\text { length, tangential } \\
(10 \mathrm{~L} / \mathrm{h}), 25^{\circ} \mathrm{C}, 8 \\
\mathrm{~L} / \mathrm{h} / \mathrm{m}^{2} \text { permeation }\end{array}$ & $\begin{array}{l}>200 \mathrm{mg} / \mathrm{h} / \mathrm{m}^{2} \\
\text { for } 24 \mathrm{~h}\end{array}$ & NR & [71] \\
\hline Tetracycline & $\begin{array}{c}T . \\
\text { versicolor } \\
\text { (Sigma- } \\
\text { Aldrich) }\end{array}$ & Free & $\begin{array}{c}100 \mu \mathrm{g} / \mathrm{mL} \\
\text { antibiotic, } 17.5 \\
\mu \mathrm{g} / \mathrm{mLlaccase}, \mathrm{pH} 7 \\
, 20^{\circ} \mathrm{C}\end{array}$ & $78 \%$ after $18 \mathrm{~h}$ & $\begin{array}{l}\text { Reduced(B.su } \\
\text { btilis) }\end{array}$ & [66] \\
\hline $\begin{array}{l}\text { Chlortetracy } \\
\text { cline, } \\
\text { doxycycline, } \\
\text { oxytetracycline }\end{array}$ & $\begin{array}{c}T \\
\text { versicolor } \\
\text { (Sigma- } \\
\text { Aldrich) }\end{array}$ & $\begin{array}{l}\text { Enzymatic } \\
\text { membrane } \\
\text { reactor }\end{array}$ & $\begin{array}{c}10 \mu \mathrm{g} / \mathrm{L} \text { antibiotics, } \\
10 \mu \mathrm{MSA}, \text { starting } \mathrm{pH} \\
6,25^{\circ} \mathrm{C}, 0.07 \mathrm{~m} / \mathrm{s} \\
\text { flow, tangential }\end{array}$ & $\begin{array}{c}85-98 \% \text { after } \\
24 \mathrm{~h}\end{array}$ & $\begin{array}{c}\text { Increased } \\
\text { (B.subtilisand } V . \\
\text { fischeri) }\end{array}$ & [39] \\
\hline
\end{tabular}




\begin{tabular}{|c|c|c|c|c|c|c|}
\hline , tetracycline & & & configuration & & & \\
\hline $\begin{array}{l}\text { Oxytetracycline } \\
\text {, tetracycline }\end{array}$ & $\begin{array}{r}\text { Cerrena } \\
\text { sp.HYB07 }\end{array}$ & $\begin{array}{r}\text { Magnetic } \\
\text { cross-linked } \\
\text { enzyme } \\
\text { aggregates }\end{array}$ & $\begin{array}{c}100 \mu \mathrm{g} / \mathrm{mL} \\
\text { antibiotic, } 40 \mathrm{U} / \mathrm{mL} \\
\text { laccase, } \mathrm{pH} 6,25^{\circ} \mathrm{C}\end{array}$ & $80 \%$ after $12 \mathrm{~h}$ & $\begin{array}{r}\text { Reduced } \\
\text { (E.coli and } B . \\
\text { licheniformis) }\end{array}$ & [66] \\
\hline \multicolumn{7}{|c|}{ QUINOLONES } \\
\hline Flumequine & $\begin{array}{c}T . \\
\text { versicolor } \\
\text { (Sigma- } \\
\text { Aldrich) }\end{array}$ & Free & $\begin{array}{l}90 \mathrm{mg} / \mathrm{L} \text { antibiotic, } \\
\quad 6 \mathrm{U} / \mathrm{mL} \\
\text { laccase, } 1.35 \mathrm{mM} \text { ABTS, } \\
\mathrm{pH} 4,39^{\circ} \mathrm{C}, 150 \mathrm{rpm}\end{array}$ & $\begin{array}{r}98 \% \\
\text { after } 2 \mathrm{~h}\end{array}$ & NR & [65] \\
\hline Ciprofloxacin & $\begin{array}{c}\text { A. } \\
\text { oryzae }\end{array}$ & Free & $\begin{array}{c}10 \mathrm{mg} / \mathrm{L} \text { antibiotic, } \\
0.02 \%(\mathrm{w} / \mathrm{v}) \text { laccase }, \mathrm{pH} 6 \\
60^{\circ} \mathrm{C}, 200 \mathrm{rpm} \text { ultrasound } \\
(75 \mathrm{~W}, 22 \mathrm{kHz}, 50 \% \text { duty } \\
\text { cycle })\end{array}$ & $\begin{array}{r}51 \% \\
\text { after } 5 \mathrm{~h}\end{array}$ & NR & {$[76]$} \\
\hline $\begin{array}{c}\text { Cinoxacin, } \\
\text { ciprofloxacin, } \\
\text { danofloxacin, } \\
\text { difloxacin, } \\
\text { enoxacin, } \\
\text { enrofloxacin, } \\
\text { flumequine, } \\
\text { marbofloxacin, } \\
\text { nalidixic acid, } \\
\text { norfloxacin, } \\
\text { ofloxacin, } \\
\text { orbifloxacin, } \\
\text { oxolinic acid, } \\
\text { pipemidic acid }\end{array}$ & $\begin{array}{l}T . \\
\text { versicolor } \\
\text { (Sigma- } \\
\text { Aldrich) }\end{array}$ & $\begin{array}{l}\text { Enzymatic } \\
\text { membrane } \\
\text { reactor }\end{array}$ & $\begin{array}{c}10 \mu \mathrm{g} / \mathrm{L} \text { antibiotics, } 10 \\
\text { or } 1,000 \mu \mathrm{M} \mathrm{SA} \text {, starting } \\
\mathrm{pH} 6,25^{\circ} \mathrm{C}, 0.07 \mathrm{~m} / \mathrm{s} \text { flow, } \\
\text { tangential configuration }\end{array}$ & $\begin{array}{c}0-84 \% \\
\text { after } 24 \mathrm{~h} \\
\text { with } 10 \mu \mathrm{M} \\
\text { SA; } 15-93 \% \\
\text { after } 24 \mathrm{~h} \\
\text { with 1,000 } \\
\mu \mathrm{M} \text { SA }\end{array}$ & $\begin{array}{l}\text { Increased (B. } \\
\text { subtilis and } V . \\
\text { ischeri) }\end{array}$ & [39] \\
\hline \multicolumn{7}{|c|}{ DIHYDROFOLATE REDUCTASE INHIBITOR } \\
\hline Trimethoprim & $\begin{array}{c}T . \\
\text { versicolor } \\
\text { (Sigma- } \\
\text { Aldrich) }\end{array}$ & $\begin{array}{l}\text { Enzymatic } \\
\text { membrane } \\
\text { Reactor }\end{array}$ & $\begin{array}{c}10 \mu \mathrm{g} / \mathrm{L} \text { antibiotic, } 1 \\
\mathrm{mM} \mathrm{SA} \text {, starting pH } 6, \\
25^{\circ} \mathrm{C}, 0.07 \mathrm{~m} / \mathrm{s} \text { flow, } \\
\text { tangential configuration }\end{array}$ & $\begin{array}{r}66.8 \% \\
\text { after } 24 \mathrm{~h}\end{array}$ & $\begin{array}{l}\text { Increased }(B . \\
\text { subtilis and } V . \\
\text { fischeri) }\end{array}$ & [39] \\
\hline Trimethoprim & $\begin{array}{c}T . \\
\text { versicolor } \\
\text { (Fluka) }\end{array}$ & $\begin{array}{l}\text { Magnetic } \\
\text { cross-linked } \\
\text { enzyme } \\
\text { aggregates }\end{array}$ & $\begin{array}{c}100 \mu \mathrm{g} / \mathrm{L} \text { antibiotic, } 1 \\
\mathrm{U} / \mathrm{mL} \text { laccase, } 0.1 \mathrm{mM} \\
\text { ABTS, pH } 7,20^{\circ} \mathrm{C}, 125 \\
\mathrm{rpm}\end{array}$ & $\begin{array}{c}47 \% \\
\text { after } 6 \mathrm{~h} ; \\
60 \% \text { after } 12 \\
\mathrm{~h}\end{array}$ & NR & [69] \\
\hline \multicolumn{7}{|c|}{ NITROIMIDAZOLE } \\
\hline Metronidazole & $\begin{array}{l}T . \\
\text { versicolor } \\
\text { (Sigma- } \\
\text { Aldrich) }\end{array}$ & $\begin{array}{l}\text { Enzymatic } \\
\text { membrane } \\
\text { reactor }\end{array}$ & $\begin{array}{l}10 \mu \mathrm{g} / \mathrm{L} \text { antibiotic, } 10 \\
\mu \mathrm{M} \mathrm{SA}, \text { starting pH } 6 \text {, } \\
25^{\circ} \mathrm{C}, 0.07 \mathrm{~m} / \mathrm{s} \text { flow, } \\
\text { tangential configuration }\end{array}$ & $\begin{array}{r}25.9 \% \\
\text { after } 24 \mathrm{~h}\end{array}$ & $\begin{array}{l}\text { Increased (B. } \\
\text { subtilis and } V . \\
\text { fischeri) }\end{array}$ & [39] \\
\hline
\end{tabular}

This is presumably due to the strong electron donating aromatic amine group in sulfonamides and the phenol group in tetracyclines, which are not found in quinolones.
However, identified tetracycline transformation intermediates suggest that the phenol group is not the primary target for laccase oxidation, and that oxygen 
addition, demethylation, water elimination reactions occur during laccase treatment [66]. For sulfonamides, increasing electro negativity of the substituents is accompanied by decreased degradation. Two sulfonamides, namely sulfapyridine and sulfathiazole, are desulfonated by laccase. Covalent cross coupling of sulfonamides is observed with laccase and mediator SA or AS [67], but not ABTS [68]. Trimethoprim has 2 amine groups and 3 methoxy groups and is usually administered in combination with sulfamethoxazole. Little [69] to over $60 \%$ [70] degradation of this antibiotic without a mediator have been reported. Furthermore, SA at 1,000 $\mu \mathrm{M}$, but not $10 \mu \mathrm{M}$, increases trimethoprim removal from 27 to $67 \%$; nearly complete elimination of sulfamethoxazole is achieved under the same conditions [71]. Some antibiotics (e.g., penicillins) are unstable in aqueous solutions, and attention should be paid to sample preservation and quantification [71]. Laccase from T.versicolor, especially the product sold by Sigma-Aldrich, is most frequently used in biodegradation studies of antibiotics as well as other trace organic contaminants. Other laccases include laccases from basidiomycetes Cerrenasp. HYB07, Echinodon tiumtaxodii, Perenniporia strain TFRI 707 and P.sanguineus, from ascomycetes Phomasp. And Myceliophthora thermophila (recombinantly expressed in Aspergillus oryzae) and from action bacteria Streptomyces ipomoeae (expressed in E. coli). Laccases immobilized by different methods have been used for antibiotic degradation; including enzymatic membrane reactors [72-74] granular activated carbon [75] silica beads oriented immobilization [67] magnetic cross linked enzyme aggregates and cell surface display [65]. In particular, enzymatic membrane reactors (gelatin ceramic membranes grafted with commercial $T$. versicolor laccase) in tetracycline degradation have been evaluated in depth with respect to membrane preparation, efficiency, kinetics, and economics [71]. Mathematical cost estimation indicates that the enzymatic process is still economically uncompetitive. Improvements should be made in terms of enzyme kinetics, reactor effective lifetime and regeneration costs. For example, a pore diameter of 1.4 $\mu \mathrm{m}$, in contrast to $0.2 \mu \mathrm{m}$, increases enzyme loading of the membrane reactor, avoids extensive membrane area, and facilitates tetracycline degradation.

Occasionally, laccases do not participate in antibiotic removal by white rot fungi; for instance, laccase was not responsible for oxy tetracycline degradation by $P$. ostreatus or $T$. versicolor or sulfamethoxazole degradation by aquatic ascomycete Phomasp. UHH 5-1-03. In these cases, other enzymes, such as cytochrome P450, may be resorted to for biodegradation. It should still be pointed out that even when extracellular laccase is not able to directly oxidize sulfamethoxazole, when a mediator is added significant removal is achieved. Laccases are also applied in combination with other processes in antibiotic treatment, such as ultrasound [76] and soil adsorption. The involvement of other processes facilitates degradation of antibiotics, e.g. quinolone antibiotics, which are recalcitrant to laccase oxidation. Laccase can also improve efficiency and stability of antibiotic removal by other organisms. When sulfamethoxazole is the transformed by non laccase producing bacteria Alcaligenes faecalis, the efficiency drops when some metabolites such as N4 acetyl sulfamethoxazole are transformed back to the parent compound. The removal efficiency does not decrease when the coculture of A.faecalis with laccase producing $P$. sanguineusis used or when cell free laccase was added to A. faecalis culture [77]. Toxicity of antibiotics after laccase treatment is commonly accessed via growth inhibition assay or bioluminescence inhibition test. Antibiotic degradation by laccase mostly leads to reduced toxicity. A good example comes from the comparison of the sulfamethoxazole transformation products and their toxicity by A. faecalis with or without exogenous laccase. N-hydroxy sulfamethoxazole (HO-SMX), a toxic and recalcitrant intermediate of sulfamethoxazole, is formed upon A. faecalis treatment. Additional laccase, on the other hand, eliminates HO-SMX along with the toxicity. However, sometimes laccase/mediator catalyzed antibiotic transformation results in even higher toxicity and this seems to frequently associate with the mediator SA [77]. It is postulated that the enhanced toxicity can be derived from oxidation of aromatic structures, especially phenols, to quinonoids.

The majority of studies on antibiotic degradation were carried out in aqueous environments, but there have been a few studies on remediation of soil, river sediment and sludge [78]. Laccase containing extract from spent mushroom compost of Pleurotus eryngii and extract containing micro capsules enhanced degradation of three tetracyclines in river sediment as well as degradation of three sulfonamides in sewage sludge. Sulfonamide antibiotics can form stable covalent bonds with humic constituents, and laccase can catalyze unreactive hydroquinone moieties in humic acid to reactive, electrophilic quionone moieties which in turn react with the antibiotic. This will affect the fate, bioactivity, and extractability of sulfonamides in soils.

\section{CONCLUSION}

This review clearly professed that laccase is one of the standout enzymes being used in peculiar areas of biotechnology such as medicine, bioremediation, 
mediator which includes improving properties of fibers, biosynthesis, energy exploitation, environmental protection, biodetection, degradation of synthetic dyes, printing and dyeing industry, biopulping in paper industry, conversion of aromatic compounds and removal of phenols which causes cancer and teratogenicity when present in waste water. In addition, it is also used in fast moving consumer goods (FMCG) as tooth-paste, mouthwash, detergent, soap, and diapers in cosmetics as deodorants; in beverage and food industry for wine and juice stabilization.

\section{ACKNOWLEDGEMENT}

The author Ram Sahay thankfully acknowledges the support of Department of Chemistry, Maharana Pratap P. G. College J. D. Gorakhpur, India.

\section{REFERENCES}

[1]. Yoshida H. Chemistry of lacquer (Urishi) part-1. J. Chem. Soc. (Tokyo) 1883; 43,472-486.

[2]. Sahay R, Yadav RSS, and Yadav KDS. Purification and Characterization of extracellular laccase secreted by Pleurotussajor-caju MTCC-141.C. J. of Biotechnology. 2008; 25: 24 (12), 2068-2073.

[3]. Dwivedi UN, Singh P, Pandey VP, Kumar A. Structure function relationship among bacterial, fungal and plant laccases, J. Mol. Cat B: Enzyme 2011; 68, 117-28.

[4]. Buddolla V, Bandi R, Avilala J, Arthala PK, and Golla N. Fungal lccase and their applications in Bioremediation, Hindawi Publishing corporation: Enzyme Research. 2014; doi:10.1155/2014/163242.

[5]. Surwase SV, Patil SA, Srinivas S, Jadhav JP. Interaction of small molecules with fungal laccase: a surface plasmon resonance based study. Enzyme.Microb. Technol.2016; 82, 110-114.

[6]. Akpinar M, Ozturk UR. Induction of fungal laccase production under solid state bio processing of new agro industrial waste and its application on dye decolorization, Biotech. 2017;7, 98.

[7]. Sahay R, Yadav RSS, and Yadav, KDS. Purification and Characterization of laccase secreted by L. lividus. Applied Biochemistry and Biotechnology. 2009; 157, 311-320.

[8]. Songulashvili G, Flahaut S, Demarez M, Trico TC, Bauvois C, Debaste F, Pen, NMJ. (2016) High yield production in seven days of Coriolopsis gallica-1184 laccase at $50 \mathrm{~L}$ scale; enzyme purification and molecular characterization. Fungal Biol. 120 (4), 481-488.

[9]. Feng, Wang, Ling Xu, Liting Zhao, Zhongyang, Ding, Haile Ma, and Norman T. Fungal Laccase Production from Lignocellulosic Agricultural Wastes by Solid-State Fermentation, Microorganisms. 2019; 7, 665.

[10]. Zheng F, Cui BK, Wu XJ, Meng G, Liu HX, Si J. Immobilization of laccase on to chitosan beads to enhance its capability to degrade synthetic dyes. Int.
Biodeterior. Biodegrad.2016; 110:69-78.

[11]. Afreen S, Anwer R, Singh RK, Fatma T. Extracellular laccase production and its optimization from Arthrospira maxima catalyzed decolorization of synthetic dyes, J. Biol. Sci. 2016; https:// doi.org /10.1016/j. sjbs. 2016.01.015.

[12]. Chenthamarakshan A, Parambayil N, Miziriya N, Soumya PS, Lakshmi MSK, Ramgopal A, Dileep A, Nambisan P. Optimization of laccase production from Maras miellus palmivorus LA1 by Taguchi method of Design of experiments, BMC Biotechnol. 2017; 17: 12.

[13]. Sahay R, Yadav RSS, Yadava Sudha, and Yadav, KDS. A Laccase of Fomes durissimus MTCC-1173 and its role in the conversion of methylbenzene to benzaldehyde. Applied Biochemistry and Biotechnology. 2012;166:563-575.

[14]. Xenakis A, Zoumpanioti M, Stamatis H. Enzymatic reactions in structured surfactant free micro emulsions. Curr. Opin. Colloid. Interface. Sci. 2016;22:41-45.

[15]. Nguyen KA, Wikee S, Lumyong S. Brief review: Ligno cellulolytic enzymes from polypores for efficien utilization of biomass. Mycosphere, 2018; 9: 1073-1088.

[16]. Zheng F, Cui BK, Wu XJ, Meng G, Liu HX, Si J. Immobilization of laccase on to chitosan beads to enhance its capability to degrade synthetic dyes.Int Biodeterior. Biodegrad.2016; 110:69-78.

[17]. Pang S, Wu Y, Zhang X, Li B, Ouyang, DingM Immobilization of laccase via adsorption onto bimodal mesoporous Zr-MOF. Process Biochem. 2016; 51 (2):229-239.

[18]. Piacquadio P, DeStefano G, Sammartino M, Sciancalepore V. Applejuice stabilization by laccase immobilized on metal chelate regenerable carriers Industrie. Delle. Bevande. 1998; 27:378-383.

[19]. Alper N, Acar J. Removal of phenolic compounds in pomegranate juices using ultrafiltration and laccase ultrafiltration combinations, Food/Nahrung.2004; 48:(3), 184-187.

[20]. Jaiswal N, Pandey VP, Dwivedi UN. Purification of a thermostable alkaline laccase from papaya (Carica papaya) using affinity chromatography, Int. J. Biol Macromol. 2015; 72:326-332.

[21]. Baldrian P. Fungal laccase occurrence and properties. FEMS Microbiol. Rev. 2006; 30:(2), 215-242.

[22]. Plácido J, Capareda S. Ligninolytic enzymes: a biotechnological alternative for bioethanol production. Bioresour. Bioprocess. 2015; 2:(1), 23.

[23]. Hakulinen N, Kiiskinen L, Kruus K, Saloheimo M, Paananen A, Koivula A, Rouvinen J. Crystal structure of a laccase from Melanocarpus albomyces with an intact trinuclear copper site, Nat. Struct. Mol. Biol. 2002; 9: 601-605.

[24]. Enguita FJ. Structural biology of fungal multicopper oxidases. In: Leitao AL (ed) Mycofactories. Bentham Science Publishers, Emirate of Sharjah, United Arab Emirates, pp. 2011; 57-72.

[25]. Felby C, Thygesen LG, Sanadi A, Barsberg S. Native lignin for bonding of fiber board evaluation of 
bonding mechanisms in boards made from laccasetreated fibers of beech (Fagussylvatica), Ind.Crops. Prod. 2004; 20:(2):181-189.

[26]. Decker H, Terwilliger N. Cops and robbers: putative evolution of copper oxygen binding proteins, J. Exp. Biol. 2000; 203:1777-1782.

[27]. Johannes C, Majcherczyk A. Natural mediators in the oxidation of polycyclic aromatic hydrocarbons by laccase mediators ystems, Appl.Environ. Microbiol. 2000; 66(2):524-528.

[28]. Terrón MC, González T, Carbajo JM, Yagüe S, AranaCuenca A,Téllez A, Dobson AD, González AE. Structural close-related aromatic compounds have different effects on laccase activity and on lcc gene expression in the ligninolytic fungus Trametes sp. I-62. Fungal Genet. Biol. 2004;41:(10), 954-962.

[29]. Leontievsky A, Myasoedova N, Pozdnyakova N, Golovleva L. Yellow laccase of Panus tigrinus oxidizes non-phenolic substrates without electron transfer mediators, FEBS. Lett. 1997; 413:(3), 446-448.

[30]. Iracheta-Cárdenas MM, Rocha-Peña MA, Galán-Wong LJ, Arévalo-Niño K, and Tovar-Herrera OE. A Pycnoporus sanguineus laccase for denim bleaching and its comparison with an enzymatic commercial formulation. J. Environ. Manage. 2016; 177:93-100.

[31]. Pozdnyakova NN, Rodakiewicz-Nowak J, Turkovskaya OV. Catalytic properties of yellow laccase from Pleurotus ostreatus D1. J. Mol. Catal. B: Enzym. 2004: 30:(1), 19-24.

[32]. Palmieri G. GiardinaP, Bianco, C Scaloni A, Capasso A, Sannia G. A novel white laccase from Pleurotus ostreatus. J. Biol. Chem. 1997; 272: (50), 31301-31307.

[33]. Zhao D, Zhang X, Cui D, Zhao M. Characterisation of a novel white laccase from the deuteromycete fungus Myrothecium verrucaria NF-05 and its decolourisation of dyes. PLoS.ONE. 2012; 7:(6), 38817.

[34]. Zhu M, Zhang G, Meng L, Wang H, Gao K, Ng T. Purification and characterization of a white laccase with pronounced dye decolorizing ability and HIV-1 reverse transcriptase inhibitory activity from Lepistanuda. Molecules. 2016; 21:(4), 415.

[35]. Ashe B, Nguyen L N, Hai FI, Lee DJ, Vande Merwe JP, Leusch FDL. et al. Impacts of redox mediator type on trace organic contaminants degradation by laccase: degradation efficiency, laccase stability and effluent toxicity, Int. Biodeterior. Biodegradation.2016; 113:169176.

[36]. Sitarz AK, Mikkelsen JD, and Meyer AS. Structure, functionality and tuning up of laccases for lignocellulose and other industrial applications. Crit. Rev.Biotechnol. 201636,70-86.

[37]. Rodríguez-Delgado M, Orona-Navar C, García-Morales R, Hernandez-Luna C, Parra R, Mahlknecht J, Ornelas-Soto N. Biotransformation kinetics of pharmaceutical and industrial micropollutants in groundwaters by a laccase cocktail from Pycnoporus sanguineus CS43 fungi. Int. Biodeter.Biodegr.2016; 108:34-41.

[38]. Daassi D, Prieto A, Zouari-Mechichi H, Martínez MJ,
Nasri M, and MechichiT.. Degradation of bisphenol A by different fungal laccases and identification of its degradation products. Int. Biodeterior. Biodegradation. 2016; 110: 181-188.

[39]. Becker D, Varela Della, Giustina S, Rodriguez-Mozaz S, Schoevaart R, BarceloD, de Cazes M.Removal of antibiotics in wastewater by enzymatic treatment with fungal laccase Degradation of compounds does not always eliminate toxicity. Bioresour. Technol. 2016; 219:500509.

[40]. Weng SS, Liu SM, and Lai HT. Application parameters of laccase-mediators ystems for treatment of sulfonamide antibiotics. Bioresour.Technol. 2013; 141: 152-159.

[41]. Fernández-Fernández M, Sanromán MÁ, and Moldes D. Recent developments and applications of immobilized laccase, Biotechnol. Adv. 2013; 31: 1808-1825.

[42]. Asgher M, Shahid M, Kamal S, and Iqbal HMN. Recent trends and valorization of immobilization strategies and ligninolytic enzymes by industrial biotechnology, J. Mol. Catal. B Enzym. 2014; 101: 56-66.

[43]. YangJ, Xu X, YangX, Ye X, and Lin J. Cross linked enzyme aggregates of Cerrena laccase: preparation, enhanced $\mathrm{NaCl}$ tolerance and decolorization of Remazol Brilliant Blue Reactive. J. Taiwan.Inst. Chem. Eng. 2016c; 65: 1-7.

[44]. Sinirlioglu ZA, Sinirlioglu D, and Akbas F. Preparation and characterization of stable cross-linked enzyme aggregates of novel laccase enzyme from Shewanell aputrefaciens and using malachite green decolorization.Bioresour.Technol.2013; 146: 807-811.

[45]. Kumar VV, Sivanesan S, and Cabana H. Magnetic crosslinked laccase aggregates bioremediation tool for decolorization of distinct classes of recalcitrant dyes, Sci. Total. Environ. 2014; 487: 830-839.

[46]. Talekar S, Joshi A, Joshi G, Kamat P, Haripurkar R, and Kambale S. Parameters in preparation and characterization of cross linked enzyme aggregates (CLEAs). RSC Adv. 2013; 3: 12485-12511.

[47]. Demarche P, Junghanns C, Nair RR, and Agathos SN. Harnessing the power of enzymes for environmental stewardship, Biotechnol. Adv. 2012; 30: 933-953.

[48]. Yang S, Hai FI, Nghiem LD, Roddick F, and Price WE. Removal of trace organic contaminants by nitrifying activated sludge and whole cell and crude enzyme extract of Trametes versicolor. Water Sci.Technol. 2013b; 67,:1216-1223.

[49]. Yadav S, and Yadav JS. Applications of ligninolytic enzymes to pollutants, water, dyes, soil, coal, paper and polymers. Environ. Tech. 2015; 13:309-318.

[50]. Martinkova L, Kotik M, Markova E, and Homolka L. Biodegradation of phenolic compounds by Basidiomycota and its phenol oxidases: a review, Chemosphere. 2016; 149:373-382.

[51]. Gasser CA, Ammann EM, Shahgaldian P, Corvini PFX. Laccases to take on the challenge of emerging organic contaminants in waste water. Appl Microbiol. Biotechnol. 2014; 98:9931-52.

[52]. Viswanath B, Rajesh B, Janardhan A, Kumar AP, and 
Narasimha G. Fungal laccases and their applications in bioremediation. Enzyme Res. 2014; 1-21.

[53]. Catherine H, Penninckx M, and Frederic D. Product formation from phenolic compounds removal by laccases: a review, Environ. Technol. Innov. 2016;5: 250-266.

[54]. Balcazar-Lopez E, Mendez-Lorenzo LH, Batista-Garcia RA, Esquivel-Naranjo U, Ayala M, Kumar VV, et al. Xenobiotic compounds degradation by heterologous expression of a Trametes sanguineus laccase in Trichoderma atroviride. PLoS ONE. 2016;11: 14-29.

[55]. Senthivelan T, Kanagara J, and Panda RC. Recent trends in fungal laccase for various industrial applications: an ecofriendly approach a review. Biotechnol. Bioprocess. Eng.2016; 21:19-38.

[56]. Zeng J, Zhu Q, Wu Y, Lin X. Oxidation of polycyclic aromatic hydrocarbons using Bacillus subtilis CotA with high laccase activity and copper independence. Chemosphere. 2016; 148:1-7.

[57]. Husain Q, and Qayyum S. Biological and enzymatic treatment of bisphenol A and other endocrine disrupting compounds: a review, Crit. Rev. Biotechnol. 2012; 33: 260-292.

[58]. Huang MT, Lu YC, Zhang S, Luo F, and Yang H. Rice (Oryzasativa) laccases involved in modification and detoxification of herbicides atrazine and isoprotur on residues in plants. J. Agric. Food. 2016; 64: 6397-6406.

[59]. Jie Yang, Wenjuan Li, Tzi BNG, Xiangzhe Deng, Juan L, and Xiuyu Ye. Laccases Production Expression Regulation and Applications in Pharmaceutical, Biodegradation. Frontiersin Microbiology. 2017; doi:10.3389/fmicb.2017.00832.

[60]. Onesios KM, Yu JT, and Bouwer EJ. Biodegradation and removal of pharmaceuticals and personal care products in treatment systems: a review. Biodegradation.2009; 20: 441-466.

[61]. Chen Y, Stemple B, Kumar M, and Wei N. Cell surface displayfungal laccase as are new able biocatalyst for degradation of persistent micro pollutants bisphenol A and sulfamethoxazole. Environ. Sci. Technol. 2016; 50: 87998808 .

[62]. Upadhyay P, Shrivastava R, and Agrawal PK. Bioprospecting and biotechnological applications of fungal laccase. Biotech. 2016;6: 15.

[63]. Mikolasch A, Manda K, Schluter R, Lalk M, Witt S, Sefeldt S. Comparative analyses of laccase catalyzed amination reactions for production of novel $\alpha$-lactam antibiotics. Biotechnol. Appl. Biochem. 2012; 59: 295-306.

[64]. Forootanfar H, and Faramarzi MA. Insights into laccase producing organisms, fermentation states, purification strategies, and biotechnological applications. Biotechnol. Prog. 2015; 31: 1443-1463.

[65]. Becker D, Rodriguez MS, Insa S, Schoevaart R, Barcelo D, Cazes M, Belleville MP, Sanchez-Marcano J, Misovic A, Oehlmann J, Wagner M. Removal of endocrine disrupting chemicals in wastewater by enzymatic treatment with fungal laccases. Org. Process. Res. Dev. 2017; $21:(4), 480-$ 91.

[66]. Llorca M, Rodríguez-Mozaz S, Couillerot O, Panigoni K, de Gunzburg J, Bayer S, et al. Identification of new transformation products during enzymatic treatment of tetracycline and erythromycin antibiotics at laboratory scale by anon-lineturbulent flow liquid-chromatography coupled to a high resolution mass spectrometer LTQOrbitrap. Chemosphere. 2015; 119: 90-98.

[67]. Shi L, Ma F, Han Y, Zhang X, Yu H. Removal of sulfonamide antibiotics by oriented immobilized laccase on $\mathrm{Fe}_{3} \mathrm{O}_{4}$ nanoparticles with natural mediators. J. Hazard. Mater. 2014; 279: 203-211.

[68]. Margot J, Copin PJ, Von-Gunten U, Barry DA, Holliger C. Sulfamethoxazole and isoproturon degradation and detoxification by a laccase-mediator system: influence of treatment conditions and mechanistic aspects. Biochem. Eng. J. 2015; 103: 47-59.

[69]. NguyenL N, Hai FI, Price WE, Leusch FD, Roddick F, Ngo $\mathrm{HH}$, et al. The effects of mediator and granular activated carbon addition on degradation of trace organic contaminants by an enzymatic membrane reactor. Bioresour. Technol. 2014b;167:169-177.

[70]. De Cazes M, Belleville MP, Mougel M, Kellner H, and Sanchez-Marcano J. Characterization of laccase-grafted ceramic membranes for pharmaceuticals degradation. J. Membr. Sci. 2015; 476: 384-393.

[71]. Nguyen LN, Vande Merwe JP, Hai FI, Leusch FD, KangJ, Price WE. Laccase syringaldehyde mediated degradation of trace organic contaminants in an enzymatic membrane reactor: removal efficiency and effluent toxicity. Bioresour. Technol. 2016b; 200: 477-484.

[72]. YangJ, YangX, Ye X, and Lin J. Destaining of Coomassie Brilliant BlueR-250-stained polyacrylamide gels with fungal laccase.Anal.Biochem.2016d ;493: 27-29.

[73]. Rodriguez-Rodriguez CE, Garcia-Galan MA, Blanquez P, Diaz-Cruz MS, Barcelo D, Caminal G. Continuous degradation of a mixture of sulfonamides by Trametes versicolor and identification of metabolites from sulfapyridine and sulfathiazole. J. Hazard. Mater. : 2012; 213 347-354.

[74]. Rahmani K, Faramarzi MA, Mahvi AH, Gholami M, Esrafili A, Forootanfar H. Elimination and detoxification of sulfathiazole and sulfamethoxazole assisted by laccase immobilized on porous silica beads. Int. Biodeterior. Biodegradation. 2015; 97:107-114

[75]. Sutar RS, and Rathod VK. Ultrasound assisted Laccase catalyzed degradation of Ciprofloxacin hydrochloride. J. Ind. Eng. Chem. 2015;31: 276-282.

[76]. Hofmann U, and Schlosser D. Biochemical and physicochemical processes contributing to the removal of endocrine disrupting chemicals and pharmaceuticals by the aquatic ascomycete Phoma sp. UHH 5-1-03. Appl. Microbiol. Biotechnol. 2016; 100: 2381-2399.

[77]. Weng SS, Ku KL, and Lai HT. The implication of mediators for enhancement of laccase oxidation of sulfonamide antibiotics. Bioresour. Technol. 2012; 113: 259-264.

[78]. Singh G, Kaur K, Puri S, and Sharma P. Critical factors affecting laccase mediated biobleaching of pulp in paper industry. Appl. Microbiol. Biotechnol. 2015; 99: 155-164 